\title{
Leminorella grimontii
}

National Cancer Institute

\section{Source}

National Cancer Institute. Leminorella grimontii. NCI Thesaurus. Code C86493.

A species of facultatively anaerobic, Gram negative, rod shaped bacteria in the phylum Proteobacteria. This species is nonmotile, hydrogen sulfide, tyrosine and $L$ arabinose positive, produces acid from dulcitol, oxidase negative, and urea, phenylalanine, and D mannose negative. L. grimontii is an emerging nosocomial pathogen, especially in immunocompromised patients. 